\title{
Single cells make the tissue
}

By pushing throughput, single-cell transcript profiling can replace markerbased sorting and bulk RNA sequencing to redefine tissues from the bottom up.

Marker genes have been closely linked to our understanding of cell types: their unique enrichment in a cell type acts as a convenient label for imaging or sorting. Like a pointillist painting, cells defined by markers are akin to dots of pure color that together form an image of a tissue's architecture and function. But this picture may be skewed. Markers offer a reductionist description of a cell that must be defined ahead of an experiment, and they can be inaccurate or depend on context.

Researchers led by Ido Amit and Amos Tanay at the Weizmann Institute in Israel scrap the need for markers by introducing massively parallel single-cell RNA sequencing (MARS-Seq) to sample and profile thousands of cells. "We were looking at a natural way to reconstruct the function of the tissue as a whole from its basic constituents," says Tanay. The results reveal the true colors of each cell in an unbiased way.

Single-cell sequencing has been used to probe tissue heterogeneity on the order of dozens of cells. But Amit notes that many cell subpopulations, such as tumor stem cells, make up a tiny fraction of the overall population. He stresses the need for robust sampling. "To understand their functional roles in vivo, you really need at least 1,000 cells to reach a $1 \%$ population," he says.

MARS-Seq is an automated 384-well plate-based assay that uses standard fluorescence-activated cell sorting and robotic liquid handling and that was designed to be user friendly. In preparing RNA for sequencing, the researchers assign unique barcodes to every transcript as well as each cell and plate. After careful normalization and filtering of sequence data to remove biases and artifacts, unsupervised analysis classifies cells into types. Clustering of expression profiles defines groups of similar cells, and the groups are used to seed a probabilistic mixture model that refines cell-type membership. The method has low technical noise, allowing data to be combined across experiments.

To achieve their ambitious goal of replacing bulk tissue sample sequenc- ing with equivalent single-cell analysis, their strategy was to pin the limiting cost to sequencing and thus ride the benefits of falling sequencing prices. MARS-Seq has already broken the barrier of $\$ 1$ per cell, enabling a routine scale of thousands of cells per experiment. The researchers estimate that given sparse sequencing and other technical limitations, only $1-2 \%$ of RNAs in a cell are currently being sampled. But the single-cell transcriptomes that they derive are still sufficiently rich to be classified into groups, and pooling RNA from subpopulations of dozens to hundreds of cells leads to accurate reconstruction of the transcriptional states within the tissue.

The teams focused their initial work on 4,000 cells from the spleen, a lymphoid organ with well-defined cell types, in order to benchmark their results. Their unsupervised analysis generated immune cell populations that matched well with known cell-type profiles. And it also found new subpopulations. "We really love this question of how you define cell type. It's one of the big promises of this technology," says Tanay. By stimulating the mouse immune system using bacterial lipopolysaccharides (LPS), they found dendritic cell subpopulations that behave quite distinctly with respect to gene expression.

The original experiment would have required treating mice and sorting cell populations, some quite rare, for individual cell sequencing and analysis-work that they estimate would take half a year. With MARS-Seq, "it's one shot, basically," says Amit. In a single one-day experiment, "you see the entire spectrum of response to LPS in a tissue, all happening in front of your eyes." In the two years it took to develop MARS-Seq, "this was one of the happiest days we had," he says.

With a robust approach to cell and tissue portraiture in hand, excitement is building to find new applications that can benefit from this unbiased resolution of cell types. Up next for the group: "many, many single cells!" says Tanay.

\section{Tal Nawy}

\section{RESEARCH PAPERS}

Jaitin, D.A. et al. Massively parallel single-cell RNAseq for marker-free decomposition of tissues into cell types. Science 343, 776-779 (2014). 